\title{
A thermodynamic counterpart of the Axelrod model of social influence: The one-dimensional case
}

\author{
Y. Gandica ${ }^{\mathrm{a}, \mathrm{b}}$, E. Medina ${ }^{\mathrm{a}}$, I. Bonalde ${ }^{\mathrm{a}}$ \\ ${ }^{a}$ Centro de Física, Instituto Venezolano de Investigaciones Científicas, Apartado 20632, \\ Caracas 1020-A, Venezuela \\ ${ }^{b}$ Center for Computational Physics, Departamento de Física, Universidade de Coimbra. \\ 3004-516 Coimbra. Portugal
}

\begin{abstract}
We propose a thermodynamic version of the Axelrod model of social influence. In one-dimensional (1D) lattices, the thermodynamic model becomes a coupled Potts model with a bonding interaction that increases with the site matching traits. We analytically calculate thermodynamic and critical properties for a 1D system and show that an order-disorder phase transition only occurs at $T=0$ independent of the number of cultural traits $q$ and features $F$. The 1D thermodynamic Axelrod model belongs to the same universality class of the Ising and Potts models, notwithstanding the increase of the internal dimension of the local degree of freedom and the state-dependent bonding interaction. We suggest a unifying proposal to compare exponents across different discrete 1D models. The comparison with our Hamiltonian description reveals that in the thermodynamic limit the original out-of-equilibrium 1D Axelrod model with noise behaves like an ordinary thermodynamic 1D interacting particle system.
\end{abstract}

\section{Introduction}

The Axelrod model [1] was proposed originally to study dissemination of cultures among interacting individuals or agents. Although the model is too simple to simulate social dynamics, the mechanisms used in the model have been recognized by social scientists as a global self-reinforcing social dynamic

\footnotetext{
${ }^{*}$ Corresponding author

Email address: ygandica@gmail.com (Y. Gandica)
} 
[2]. It is a fact that the more culturally similar the people, the greater the chance of interaction between them, and that interaction increases their similarity [3]. These are the premises of the model.

More explicitly, the Axelrod model considers that an agent located at the $i^{\text {th }}$ site of a lattice is defined by a set of $F$ cultural features (e.g., religion, sports, politics, etc.) represented by a vector $\sigma_{i}=\left(\sigma_{i 1}, \sigma_{i 2}, \ldots, \sigma_{i F}\right)$. Each feature $\sigma_{i k}$ can take integer values in the interval $[1, q]$, where $q$ defines the cultural traits allowed per feature and measures the cultural variability in the system. There are $q^{F}$ possible cultural states. The model's dynamics is as follows: (1) Choose randomly two nearest neighbor agents $i$ and $j$, then (2) calculate the number of shared features between the agents $\ell_{i j}=\sum_{k}^{F} \delta_{\sigma_{i k}, \sigma_{j k}}$. If $0<\ell_{i j}<F$, then (3) pick up randomly a feature $k$ such that $\sigma_{i k} \neq \sigma_{j k}$ and with probability $\ell_{i j} / F$ set $\sigma_{i k}=\sigma_{j k}$. These time steps are iterated and the dynamics stops when a frozen state is reached; i.e., either $\ell_{i j}=0$ or $\ell_{i j}=F, \forall i, j$. A cluster is a set of connected agents with the same state. Monocultural or ordered phases are composed of a cluster of the size of the system where $\ell_{i j}=F, \forall i, j$. Multicultural or disordered phases consist of two or more clusters.

One of the main features of this model is a change of behavior at a value $q_{c}$ from a monocultural state, where all agents share the same cultural features, to a multicultural state, where individuals mostly have their own features [4]. This change can be characterized by an order parameter $\phi$ that is usually defined as the average size of the largest cultural cluster $C_{\max }$ normalized by the total number of agents $N$ in the system; $\phi=C_{\max } / N$. In the monocultural (ordered) state $\phi \rightarrow 1$ and in the multicultural (disordered) state $\phi \rightarrow 0$.

The insertion of additional ingredients in the model, like an external field or mass media, yields interesting nontrivial consequences in the system [5, 6, 7]. The main limitation of the model seems to be that the system always converges to absorbent states, a situation that clearly does not occur in society. Some variants of the model relax this tendency by introducing noise into the system [8, 9, 10]. If the noise rate is small, the system reaches only monocultural states. However, if the noise rate is above a size-dependent critical value, a polarized state is sustained [8, 9, 10]. Klemm et al. [8, 9] associated the monocultural (multicultural) states with stable (unstable) equilibria.

Until now, in the sociophysics field the global dynamics of social systems have been usually studied by postulating a series of rules that at the 
end lead to out-of-equilibrium behaviors, such as absorbent states. This approach often uses statistical mechanics concepts -temperature, critical phase transition, applied magnetic field, among others- without formal definitions. Langevin-type approaches have been proposed to study the collective phenomena of the social systems in terms of their microscopic constituents and their interactions [11]. Little attention has been paid to this approach in which the system can be modeled in a Hamiltonian formulation, whereupon equilibrium and nonequilibrium behaviors can be explored [12, 13]. Such a Hamiltonian description also allows for the understanding of the meaning of social variables in the context of statistical mechanics.

Here, we develop a Hamiltonian version of the Axelrod model of social influence. Our Hamiltonian captures the local interactions of the original model. With the aim of finding a possible thermodynamic role of the parameters $F, q$, and $q_{c}$, our model, henceforth called thermodynamic Axelrod, uses the number of shared features $\ell_{i j}$ of the Axelrod model to construct a new Hamiltonian distinguishable from the 1D $F$-parallel-layer Potts models in that the interaction strength between agents increases with $\ell_{i j}$. This feature of the interaction precipitates ordering preempting fluctuations. In the thermodynamic Axelrod model $F$ is related to the coupling energy of the system and $q$ has the same meaning as in the Potts model.

Although it is usually argued that the Axelrod model is an out-of-equilibrium model, this fact, taken as obvious, has never been demonstrated in the literature. In Sect. II we demonstrate that the standard Axelrod model does not satisfy the detailed balance condition. In Sect. III we analytically calculate the main thermodynamic functions for our model. For the critical behavior analysis (Sect. IV-b) we make an unifying proposal to compare exponents across different 1D discrete models, since current definitions depend on model details. In Sect. $\mathrm{V}$ we state the consequences of our study over the transitions driven by noise in the original Axelrod model. We discuss the implications of a thermodynamic society in Sect. VI and in Sect. VII we present our conclusions.

\section{Axelrod model: Out of equilibrium}

Before we introduce the thermodynamic version of the Axelrod model, here we demonstrate that the original version does not satisfy equilibrium conditions by showing that detailed balance is violated. 
Let a link between two sites $i$ and $j$ be of type $n$ if they share $n$ components $\left(\ell_{i j}=n\right), P_{n}$ be the probability that the system is in a state with links of type $n$, and $W_{n m}$ be the transition probability per unit time from a state with type- $n$ to one with type- $m$ links. $W_{n m}$ being time independent. Since in the dynamics of the Axelrod model a feature $k$ is changed with probability $\ell_{i j} / F$ to make two sites have one more component in common $\left(\sigma_{i k}=\sigma_{j k}\right)$, we have

$$
W_{n m}=\left\{\begin{array}{cll}
\frac{n}{F} & \text { for } & m=n+1 \\
0 & & \text { otherwise }
\end{array}\right.
$$

The detailed balance relation implies that [14]

$$
W_{n m} P_{m}=W_{m n} P_{n} \quad \forall n, m .
$$

In the Axelrod model Eq. (2) cannot be satisfied since one side or the other is always zero according to Eq. (11). This feature of the model introduces some very strong constraints into both the evolution and the time-independent states of interactions that emphasize nonequilibrium; i.e. i) completely different individuals do not interact, ii) individuals conform once they have modified their cultural profile, and iii) individuals that are alike, that interact, increase their similarity at interaction. These rules yield absorbing states, the most salient nonequlibrium feature of the Axelrod model.

The thermodynamic model we propose relaxes all of the previous constraints, while preserving similarity, increasing interactions, and introducing fluctuations controlled by a temperature parameter. These conditions permit arriving at a dynamical equilibrium state in the regular sense of statistical mechanics. On the other hand, we are interested in evaluating whether behaviors reported in nonequilibrium network models survive in a thermodynamic driven scenario.

\section{Thermodynamic Axelrod model}

To reproduce the interaction rule of the Axelrod model, in which the interaction probability is proportional to the number of shared features, the Hamiltonian is defined as

$$
\mathcal{H}=-\sum_{k=1}^{F} \sum_{i j}^{N}\left(J_{i j} \delta\left(\sigma_{i k}, \sigma_{j k}\right)+\frac{\mu H}{2}\left[\delta\left(\sigma_{i k}, H_{k}\right)+\delta\left(\sigma_{j k}, H_{k}\right)\right]\right),
$$


with the interaction factor

$$
J_{i j}=\sum_{n=1}^{F} J \delta\left(\sigma_{i n}, \sigma_{j n}\right) .
$$

The delta function captures the local interactions of the original model, inasmuch as the interaction strength between agents increases with the number of shared features $\ell_{i j}$. In this way our Hamiltonian system takes into account both the tendency of individuals to become more similar when they interact, namely social influence (like the voter model), and the greater tendency to interact with individuals which are more similar, namely homophyly (specific of the Axelrod model). $H_{k}$ works as an applied magnetic field that (a) can point in one of the Potts-model-like directions $k$, (b) can take values $1, \ldots, q$, and (c) has an energy weight proportional to the magnitude $H . \mu$ is the magnetic-like moment per agent. $\sigma_{i k}=1, \ldots, q$ specifies each of the $F$ variables $\left(\sigma_{i 1}, \sigma_{i 2}, \ldots, \sigma_{i F}\right)$ of the agent $\sigma_{i}$ at the $i$ th lattice site. $N$ is the size of the system. The second term in the Hamiltonian is symmetrized for convenience.

The Hamiltonian in Eq. (3) is evidently inspired on the Potts model, with the significant distinction that the interaction factor $J_{i j}$ always depends on the global state of the F-vector and not on the state of the particular Potts variable. This is a somewhat rare type of Hamiltonian interaction, in a sense similar to a nonlinear sigma model where a vector interaction occurs subject to normalization of the interacting vectors [15]. Our model on a 1D lattice is like an F-coupled-layer Potts model. It is then a quasi $1 \mathrm{D}$ system, thus some signatures of the two-dimensional (2D) Potts model are expected as crossovers.

We consider a 1D chain of $N$ sites occupied by $q^{F}$-valued agents and use the transfer matrix method to compute the relevant physical properties. For periodic boundary conditions $\sigma_{(N+1) k}=\sigma_{1 k}$, the partition function corresponding to the above Hamiltonian can be expressed as 


$$
\begin{aligned}
Z= & \sum_{\sigma_{1}} \sum_{\sigma_{2}} \cdots \sum_{\sigma_{N}} \prod_{i=1}^{N} \exp \left[\sum _ { k = 1 } ^ { F } \left(\beta J_{i(i+1)} \delta\left(\sigma_{i k}, \sigma_{(i+1) k}\right)\right.\right. \\
& \left.\left.+\frac{\beta \mu H}{2}\left[\delta\left(\sigma_{i k}, H_{k}\right)+\delta\left(\sigma_{(i+1) k}, H_{k}\right)\right]\right)\right] \\
= & \operatorname{Tr}\left[W^{N}\right] .
\end{aligned}
$$

Here, $\beta=1 / k_{B} T$ and we introduced a $q^{F} \times q^{F}$ transfer matrix $W$ with elements

$$
\begin{aligned}
\left\langle\sigma_{i}|W| \sigma_{i+1}\right\rangle= & \exp \left[\sum _ { k = 1 } ^ { F } \left(\beta J_{i(i+1)} \delta\left(\sigma_{i k}, \sigma_{(i+1) k}\right)\right.\right. \\
& \left.\left.+\frac{\beta \mu H}{2}\left[\delta\left(\sigma_{i k}, H_{k}\right)+\delta\left(\sigma_{(i+1) k}, H_{k}\right)\right]\right)\right] .
\end{aligned}
$$

The eigenvalues $\lambda_{j}$ of the transfer matrix are determined from the solution of the secular equation Det $|W-\lambda E|=0$. Then, the partition function can be written as

$$
Z=\lambda_{1}^{N}+\lambda_{2}^{N}+\cdots \lambda_{q^{F}}^{N}=\lambda_{\max }^{N}\left(1+\frac{\lambda_{1}^{N}}{\lambda_{\max }^{N}}+\cdots+\frac{\lambda_{q^{F}}^{N}}{\lambda_{\max }^{N}}\right)
$$

where $\lambda_{\max }$ is the largest eigenvalue. In the thermodynamic limit $(N \rightarrow$ $\infty), Z \cong \lambda_{\max }^{N}$. Then, a standard procedure [16] can be followed to obtain thermodynamic and critical properties.

\section{Case $F=2, q=2$}

Here, we present explicitly the simplest nontrivial case $\mathrm{F}=2, \mathrm{q}=2$, as an illustration. Analytical calculations were performed for higher values of $\mathrm{F}$ and $\mathrm{q}$ but full expressions are too lengthy. The transfer matrix takes the form

$$
W=\left(\begin{array}{cccc}
e^{2 \beta \mu H+4 \beta J} & e^{\frac{3}{2} \beta \mu H+\beta J} & e^{\frac{3}{2} \beta \mu H+\beta J} & e^{\beta \mu H} \\
e^{\frac{3}{2} \beta \mu H+\beta J} & e^{\beta \mu H+4 \beta J} & e^{\beta \mu H} & e^{\frac{1}{2} \beta \mu H+\beta J} \\
e^{\frac{3}{2} \beta \mu H+\beta J} & e^{\beta \mu H} & e^{\beta \mu H+4 \beta J} & e^{\frac{1}{2} \beta \mu H+\beta J} \\
e^{\beta \mu H} & e^{\frac{1}{2} \beta \mu H+\beta J} & e^{\frac{1}{2} \beta \mu H+\beta J} & e^{4 \beta J}
\end{array}\right)
$$


The largest eigenvalue for this matrix is

$$
\begin{aligned}
\lambda_{\max }= & \frac{1}{3}\left(e^{4 \beta J+\beta \mu H}+e^{4 \beta J+2 \beta \mu H}+e^{4 \beta J}+e^{\beta \mu H}\right)-\frac{2^{1 / 3} a}{3\left[b+\sqrt{4 a^{3}+b^{2}}\right]^{1 / 3}} \\
& +\frac{1}{32^{1 / 3}}\left[b+\sqrt{4 a^{3}+b^{2}}\right]^{1 / 3}
\end{aligned}
$$

where

$$
\begin{aligned}
a= & -6 e^{2 \beta J+\beta \mu H}+e^{4 \beta J+\beta \mu H}+e^{8 \beta J+\beta \mu H}-2 e^{4 \beta J+2 \beta \mu H}-6 e^{2 \beta J+3 \beta \mu H} \\
& +e^{4 \beta J+3 \beta \mu H}+e^{8 \beta J+3 \beta \mu H}-e^{8 \beta J+4 \beta \mu H}-e^{8 \beta J}-4 e^{2 \beta \mu H} \\
b= & 18 e^{6 \beta J+\beta \mu H}-3 e^{8 \beta J+\beta \mu H}-3 e^{12 \beta J+\beta \mu H}+18 e^{2 \beta J+2 \beta \mu H}+6 e^{4 \beta J+2 \beta \mu H} \\
& +18 e^{6 \beta J+2 \beta \mu H}-6 e^{8 \beta J+2 \beta \mu H}-6 e^{12 \beta J+2 \beta \mu H}+108 e^{2 \beta J+3 \beta \mu H}-12 e^{4 \beta J+3 \beta \mu H} \\
& -72 e^{6 \beta J+3 \beta \mu H}+18 e^{8 \beta J+3 \beta \mu H}+14 e^{12 \beta J+3 \beta \mu H}+18 e^{2 \beta J+4 \beta \mu H}+6 e^{4 \beta J+4 \beta \mu H} \\
+ & 18 e^{6 \beta J+4 \beta \mu H}-6 e^{8 \beta J+4 \beta \mu H}-6 e^{12 \beta J+4 \beta \mu H}+18 e^{6 \beta J+5 \beta \mu H}-3 e^{8 \beta J+5 \beta \mu H} \\
- & 3 e^{12 \beta J+5 \beta \mu H}+2 e^{12 \beta J+6 \beta \mu H}+2 e^{12 \beta J}-16 e^{3 \beta \mu H}
\end{aligned}
$$

The free energy, magnetization, magnetic susceptibility per particle, and specific heat are given in terms of $\lambda_{\max }$ :

$$
\begin{gathered}
\mathcal{F}=-k_{B} T \ln \lambda_{\max } \\
M=-\frac{\partial \mathcal{F}}{\partial H}=\frac{k_{B} T}{\lambda_{\max }} \frac{\partial \lambda_{\max }}{\partial H} \\
\chi=\frac{\partial M}{\partial H}=\frac{\partial}{\partial H}\left(\frac{k_{B} T}{\lambda_{\max }} \frac{\partial \lambda_{\max }}{\partial H}\right), \\
C=-T \frac{\partial^{2} \mathcal{F}}{\partial T^{2}}=2 \frac{k_{B} T}{\lambda_{\max }} \frac{\partial \lambda_{\max }}{\partial T}+k_{B} T^{2} \frac{\partial}{T}\left(\frac{1}{\lambda_{\max }} \frac{\partial \lambda_{\max }}{\partial T}\right) .
\end{gathered}
$$

Figures 1-4 show plots of the magnetization, magnetic susceptibility, and specific heat in terms of $k_{B} T / J$ and $\mu H / k_{B} T$. The magnetization (Fig. 1) goes to 0 as $H \rightarrow 0$ at any finite $T$. At $T=0$, the magnetization saturates 

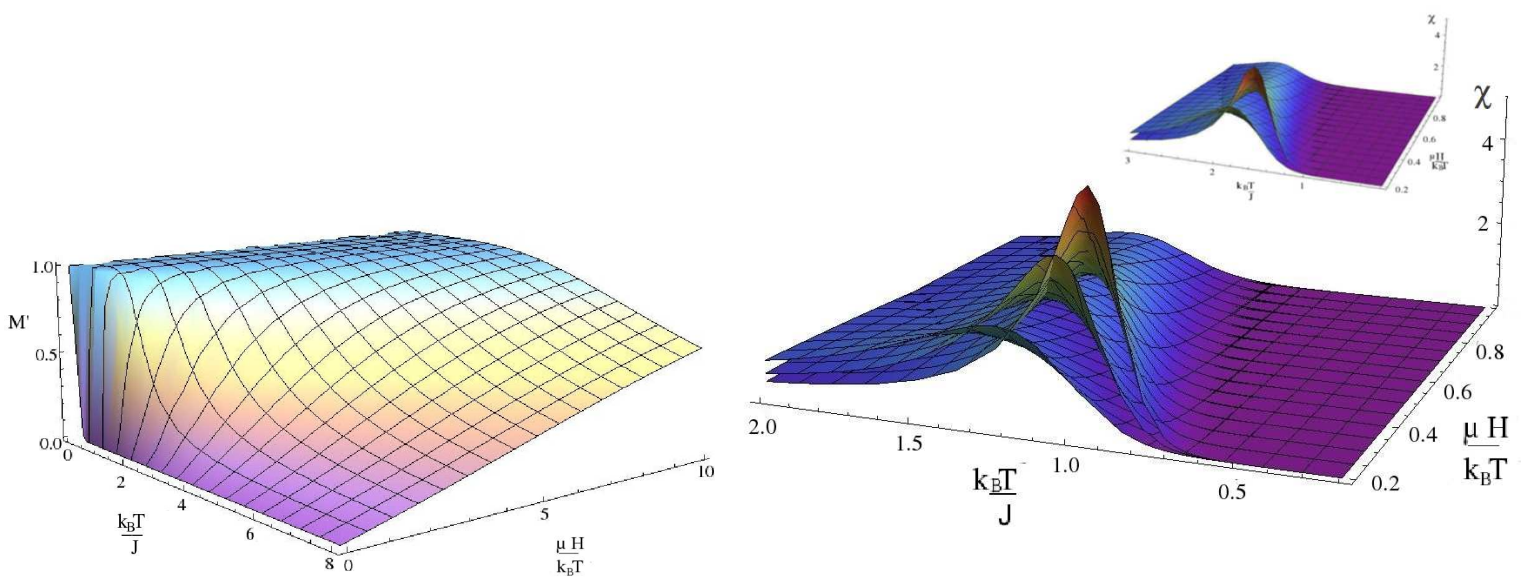

Figure 1: Magnetization of the 1D thermodynamic Figure 2: Susceptibility of the 1D thermodynamic Axelrod model for the case $F=2$ and $q=2$. Axelrod model for the case $F=2$ with $q=2,3$ and 4 . In the inset we show the case $F=3$ with $q=2$ and 3 .
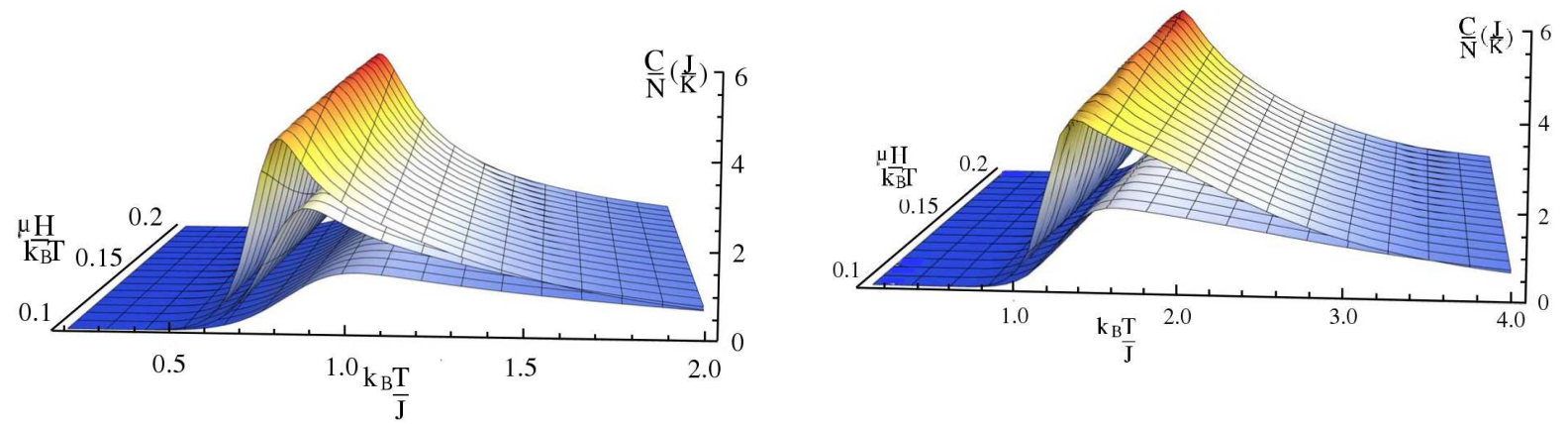

Figure 3: Specific heat of the 1D thermodynamic Figure 4: Specific heat of the 1D thermodynamic Axelrod model for the case $F=2$ and $q=2,3$ Axelrod model for the case $F=3$ and $q=2$ and and 4 . $q=3$. 
to its maximum value for any $H$. This implies a spontaneous transition to an ordered state only at $T=0$. When $T$ is finite, the magnetization saturates to its maximum only at large $H$. Thus, the changes in the internal space dimensions of the model do not affect the scenario expected for one dimension. The increased fluctuations derived from the greater dimensions of the internal vector space destroy order except at $T=0$, as would be expected.

The susceptibility diverges as $T \rightarrow 0$ and $H \rightarrow 0$. As we will derive analytically in the following section, the divergence is independent of the values $F$ and $q$ and corresponds to the Potts class. On the other hand, the nonuniversal prefactors become larger as the value of $q$ increases, as can be seen in Fig,2. The farther from the singularity in the $T$ direction, the faster high- $q$ susceptibilities die out. The opposite is true in the $H$ direction. This is the scenario in both $F=2$ and $F=3$.

As expected, the temperature of the maximum value of the specific heat $C$ (Schottky anomaly) increases for larger $F$ values (Fig. 3 and Fig. 4). As a result of the coupling, the ordered system is more robust and requires more energy to be destroyed. For a fixed $F$ value, $C$ depends only on the external field at different values of $q$. Since the specific heat is proportional to the amount of energy per agent the system can absorb, as $q$ increases the number of accessible states is greater and, therefore, a higher external field is required to orient the agents in the same direction. The dependence of $C$ with temperature is through the the gap of the system (difference between the ground state and the first excited state), which does not vary with $q$ (see Eq. (23) below). All these properties behave qualitatively as they do in the Ising and Potts models on 1D lattices.

The specific heat can be regarded as the resistance of the society to increase the fluctuation average in posture. For lower values of $q$ it is easier to change the average size of fluctuations, because there are less options for disagreement in the system. The gap, which increases with $F$, is the energy necessary for the system to break similarity bonds between individuals in the ground state.

The susceptibility is the magnetic response of the system to external field. In the thermodynamic society, it represents the relative ease of social alignment to the mass media. As thermal fluctuations decrease, a weaker mass media makes for a larger effect. This can be seen from the fact that as $q$ increases the system magnetizes more easily at smaller fields.

In terms of the thermal society of agents, the above results imply that, as 
a consequence of the periodic boundary conditions, the fluctuating postures will always outweigh the benefits of agreement so that spontaneous cultural uniformity does not occur. Uniformity can occur, however, for any finite mass media.

\subsection{Spatial correlations}

We now calculate the two-point correlation function

$$
G(i, i+j)=\left\langle\sigma_{i} \sigma_{i+j}\right\rangle-\left\langle\sigma_{i}\right\rangle\left\langle\sigma_{j}\right\rangle
$$

using the transfer matrix method [17]. The first term is given by

$$
\left\langle\sigma_{i} \sigma_{i+j}\right\rangle=\frac{1}{Z} \operatorname{tr}\left[A W^{j} A W^{N-j}\right],
$$

where $A=\sum_{\sigma_{i}}\left|\sigma_{i}\right\rangle \sigma_{i}\left\langle\sigma_{i}\right|$. In the original space for $F=2$ and $q=2$, the state matrix

$$
A=\left(\begin{array}{llll}
1 & 0 & 0 & 0 \\
0 & 2 & 0 & 0 \\
0 & 0 & 2 & 0 \\
0 & 0 & 0 & 4
\end{array}\right)
$$

Following a standard procedure, we evaluate Eq. (15) in a basis where $W$ is diagonal. For $H=0$, the unitary matrix that diagonalizes $W$ is

$$
P=\left(\begin{array}{cccc}
-1 & 0 & 1 & 1 \\
0 & -1 & -1 & 1 \\
0 & 1 & -1 & 1 \\
1 & 0 & 1 & 1
\end{array}\right)
$$

The evaluation yields

$$
\begin{aligned}
\left\langle\sigma_{i} \sigma_{i+j}\right\rangle= & \frac{1}{Z} \operatorname{tr}\left[\left(\begin{array}{cccc}
\frac{5}{2} & 0 & \frac{3}{2} & \frac{3}{2} \\
0 & 2 & 0 & 0 \\
\frac{3}{4} & 0 & \frac{9}{4} & \frac{1}{4} \\
\frac{3}{4} & 0 & \frac{1}{4} & \frac{9}{4}
\end{array}\right)\left(\begin{array}{cccc}
\lambda_{1}^{j} & 0 & 0 & 0 \\
0 & \lambda_{2}^{j} & 0 & 0 \\
0 & 0 & \lambda_{3}^{j} & 0 \\
0 & 0 & 0 & \lambda_{4}^{j}
\end{array}\right)\left(\begin{array}{cccc}
\frac{5}{2} & 0 & \frac{3}{2} & \frac{3}{2} \\
0 & 2 & 0 & 0 \\
\frac{3}{4} & 0 & \frac{9}{4} & \frac{1}{4} \\
\frac{3}{4} & 0 & \frac{1}{4} & \frac{9}{4}
\end{array}\right)\right. \\
& \left.\times\left(\begin{array}{cccc}
\lambda_{1}^{N-j} & 0 & 0 & 0 \\
0 & \lambda_{2}^{N-j} & 0 & 0 \\
0 & 0 & \lambda_{3}^{N-j} & 0 \\
0 & 0 & 0 & \lambda_{4}^{N-j}
\end{array}\right)\right] \\
= & \frac{81}{16}+\frac{9}{8}\left(\frac{\lambda_{1}}{\lambda_{4}}\right)^{j} .
\end{aligned}
$$


Here, $\lambda_{4}$ and $\lambda_{1}$ are the largest and the second largest eigenvalues, respectively. The evaluation was performed in the thermodynamic limit.

The average of the agent at site $i,\left\langle\sigma_{i}\right\rangle$, is evaluated in the same manner:

$$
\left\langle\sigma_{i}\right\rangle=\frac{1}{Z} \operatorname{tr}\left[A W^{N}\right]=\frac{9}{4} .
$$

Then,

$$
G(i, i+j)=\frac{9}{8}\left(\frac{\lambda_{1}}{\lambda_{4}}\right)^{j} \approx e^{-j \ln \left(\lambda_{4} / \lambda_{1}\right)} \equiv e^{-j / \xi} .
$$

The correlation function has the same form as in the Ising and Potts models [18, 17], with the correlation length

$$
\xi=\frac{1}{\ln \left(\lambda_{4} / \lambda_{1}\right)} .
$$

Since $\lambda_{1}=-1+e^{4 J / k_{B} T}$ and $\lambda_{4}=1+2 e^{J / k_{B} T}+e^{4 J / k_{B} T}$, the correlation length becomes

$$
\xi=\frac{1}{\ln \left[\left(1+2 e^{J / k_{B} T}+e^{4 J / k_{B} T}\right) /\left(-1+e^{4 J / k_{B} T}\right)\right]} .
$$

As in Ising and Potts models, for $H=0$ the two largest eigenvalues become degenerate at $T=0$, which leads to a divergence of the correlation length and, therefore, to a zero-temperature phase transition.

In social terms, the correlation length measures the distance at which there are relations between agents beyond their own mean values. This is a causal or influence relationship in the sense that changing the opinions in one place generates an influence that causes change up to the correlation length. This influence operates through local interactions. In terms of social influence the existence of a correlation length invokes a limit to the propagation of influence. A return force is a cost for producing fluctuations and this cost avoids the propagation of fluctuations beyond a certain distance. When no return force is present (critical point) fluctuations diverge and influence runs over the whole society at all scales.

\subsection{Critical exponents}

To get the critical behavior of the thermodynamic properties one needs to evaluate them near the transition temperature $T_{c}$. For $1 \mathrm{D}$ models, including the present one, the transition occurs at $T_{c}=0$ with exponential singularities [16]. In this case, the usual reduced temperature $t=\left(T-T_{c}\right) / T_{c}$ is 
inappropriate. A different critical point approach parameter $t=e^{-\Delta / k_{B} T}$ [16] is required to convert the exponential singularities in $T$ into power-law singularities in $t$. The constant $\Delta$ has so far been taken arbitrarily.

Here, we propose that $\Delta$ is given by half the energy difference between the ground state and the first excited state of the system. In this way, $\Delta$ eliminates from the value of the exponent any nonuniversal features, which will be present otherwise. This convention correctly unifies, independently of the interaction strength, the Ising and Potts exponents. For the 1D Ising model the energy difference between the ground state (say, all spins aligned up) and the first excited state (one spin aligned opposite to the others) is $4 \mathrm{~J}$; then, for this case $\Delta=2 J$. This agrees with the choice $p=2$ in $t=e^{-p J / k_{B} T}$ [16] to bring together the exponents of the 1D discrete-symmetry models.

For the Potts and thermodynamic Axelrod models, the energy of the ground state is $-J N F^{2}$, whereas the energy of the first excited state is

$$
-J\left[(N-2) F^{2}+2(F-1)^{2}\right] .
$$

Then,

$$
\Delta=J(2 F-1) .
$$

In the case of the Potts model, $F=1$ and $\Delta=J$. This value of $\Delta$ yields critical exponents of the Potts model that agree with those of the Ising model [18]. For the thermodynamic Axelrod model, $F=2$ and $\Delta=3 J$.

We now can estimate the critical exponents of our model for the case $F=2$ and $q=2$. We define $h=\mu H / k_{B} T$ and have $t=e^{-3 J / k_{B} T}$. For $H=0$ and $t \rightarrow 0$ the singular part of the free energy, Eq. (10), for a zerotemperature transition [16] becomes

$$
f(t)=\frac{\mathcal{F}+4 N J}{N k_{B} T} \sim t .
$$

Since $f \sim t^{2-\alpha}, \alpha=1$. In the same limit, the magnetization, Eq. (11), is

$$
m(t)=\frac{M}{N \mu} \sim 1
$$

This means from $m \sim t^{\beta}$ that $\beta=0$. Now, for $t=0$ and $H \rightarrow 0$, the magnetization becomes

$$
m(h)=\frac{M}{N \mu} \sim 1
$$


Since $m \sim h^{1 / \delta}$, the exponent $\delta \rightarrow \infty$. The low-field susceptibility is obtained from Eq. (12)

$$
\chi_{0}(t)=\frac{\chi k_{B} T}{N \mu^{2}} \sim \frac{1}{2} t^{-1} .
$$

The susceptibility should go as $t^{-\gamma}$, then $\gamma=1$. The specific heat, Eq. (13), for $H=0$ and $t \rightarrow 0$ is

$$
c(t)=\frac{C k_{B} T^{2}}{N J^{2}} \sim-\frac{4}{3} t
$$

Then, from $C \sim t^{\alpha}$ one gets $\alpha=1$. Finally, from Eq. (22) the correlation lenght

$$
\xi(t) \sim \frac{1}{2} t^{-1}
$$

The correlation length goes as $t^{-\nu}$; then, $\nu=1$.

In conclusion, the differences in the internal space dimensionality $(F=2)$ and interaction strengths of our model do not alter the Ising universality class. We performed the same calculation for higher values of $F$ and $q$, obtaining the same results. All the results presented in this section indicate that the $1 \mathrm{D}$ thermodynamic Axelrod model exhibits a phase transition at $T=0$.

\section{Thermodynamic and social Axelrod models: a comparison}

We first compare our results with those obtained for the original Axelrod model without the inclusion of any effect. In this case, a monoculturalmulticultural (order-disorder) transition is observed at a threshold value $q_{c}$. Figure 5 depicts the analytical results for the temperature dependence of the order parameter for $F=2$ and $q=2,3,4,5$. For comparison, we also show curves for $F=3$ and $q=2,3$. A monotonic and smooth dependence on $q$ is observed for $F=2$, indicating no phase transition at finite temperature. For both values of $F$ the order parameter behaviors are similar to those expected in the Potts model [18]. We note that for the analyzed values of $F$, as $q$ increases the transition becomes gradually sharper. The data of Fig. 5 were obtained, just to be able to perform the numerical calculations, at the very small field of $0.005 k_{B} T / \mu$.

The Axelrod model has also been studied with the addition of noise (cultural drift) [8, 9, 10]. By analyzing the equilibrium configurations and their stability, it was found that below a critical value of the noise rate $r_{c}$ (noise 


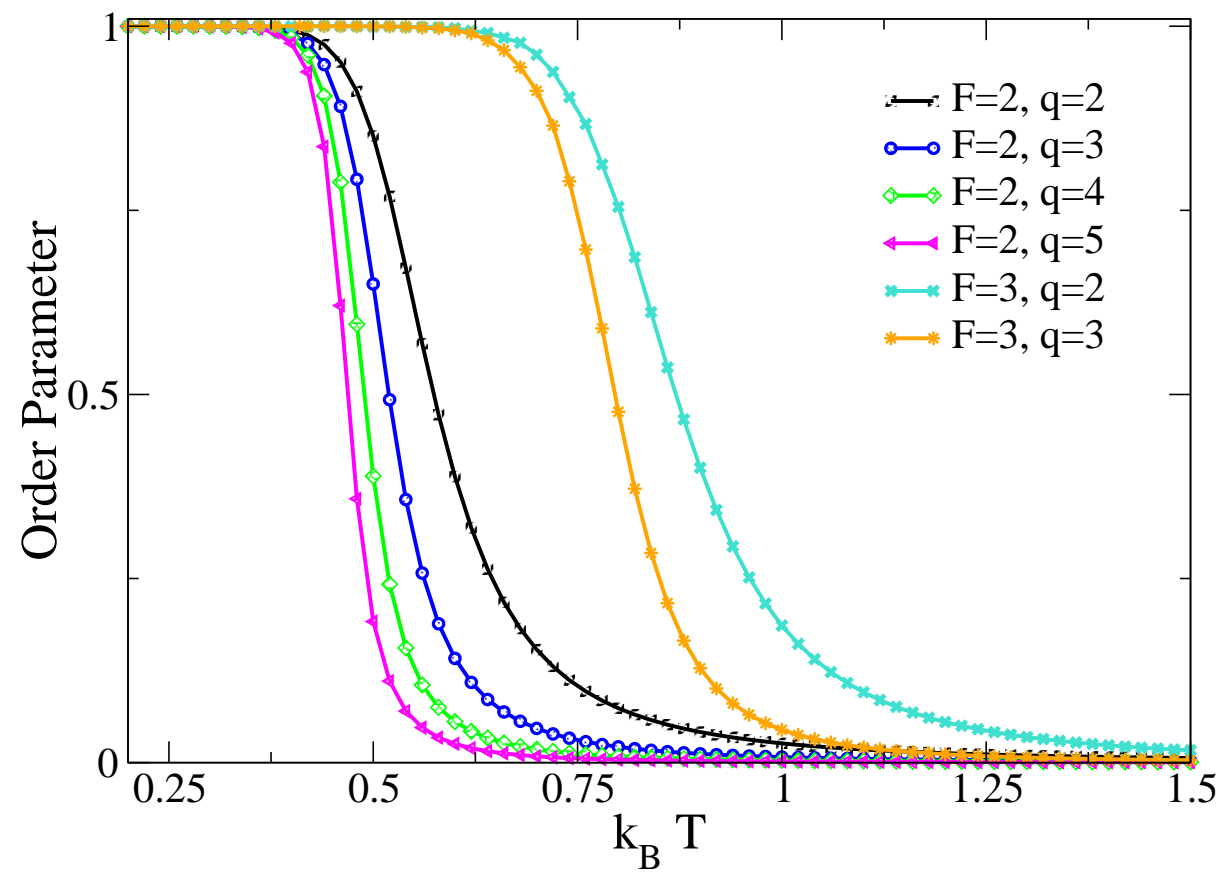

Figure 5: Order parameter of the thermodynamic Axelrod model for $H=0.005$. The group of curves to the left correspond to $F=2$ and the ones to the right to $F=3$. No anomaly is observed as $q$ varies from 2 to 5 for the $F=2$ case. In the nonequilibrium Axelrod model a crossover from an ordered to a disordered state is seen below $q=5$ in the $1 \mathrm{D}$ case [19]. 
is introduced as a perturbation of a single agent at certain times during the dynamics) the initially multicultural population converges to a monocultural one, whereas for $r>r_{c}$ the system moves to a multicultural state [8]. The threshold value $r_{c}$ depends on the system size and is almost independent of q. In $1 \mathrm{D} r_{c} \sim 1 / N^{2}[8,10]$, thus in the limit $N \rightarrow \infty$, the value $r_{c} \rightarrow 0$, implying that in this condition there is no phase transition and that for any finite $r$ the system converges to a multicultural state [8, 10]. This behavior is analog to the one found in the thermodynamic Axelrod model, in which for any finite $T$ the system goes to a multicultural state. The polarized state for any $r>0$ is equivalent to the zero-magnetization phase for any finite $T$ in discrete-symmetry systems such as the present one. The noise variable $r$ corresponds to temperature $T$.

The implications of the thermodynamic limit $(N \rightarrow \infty)$ for the noisy Axelrod model are known [8, 9, 10]. Our study, focused on the equilibrium approach, underlines a possible connection between the thermodynamic model and the nonequlibrium noisy Axelrod model. Universal behavior is equivalent once one equates noise and thermal fluctuations. It is worthwhile to study then the present Hamiltonian model in higher dimensions to verify the latter assertion. It is also interesting to see whether the energy tendency to increase similarity of agents can change the universality class from that of Potts.

Regarding the effect of a magnetic field; at $T=0$, a $1 \mathrm{D}$ thermodynamic system is always magnetized for any value of the applied field. Even though a direct comparison is only qualitative, it is worth mentioning that in the original Axelrod model an external field (mass media) gives rise to related behavior in a 2D system. By using, as the order parameter, the average fraction of cultural clusters $g=\left\langle N_{g}\right\rangle / N$, where $N_{g}$ is the number of clusters formed in the final state, it was suggested that, in 2D, mass media can induce a multicultural state when its strength is above a certain threshold [5, 6]. This was also shown with the order parameter $\phi$ [7]. Using the order parameter $g$, Peres and Fontanari [20] showed that in 2D the effect of mass media on the Axelrod model always (for any value of $H$ ) displays a tendency toward cultural diversity when $N \rightarrow \infty$. That is to say, no order-disorder transition is observed with $g$ in such limit. On the other hand, when the normalized size of the largest cluster $\phi$ is used as the order parameter (the one applied in our work) in the original Axelrod model, the results indicate that the effect of mass media, inducing a multicultural phase, persists in the limit $N \rightarrow \infty$ [21]. Hence, it would be of interest to study the 2D thermodynamic Axelrod 
model with both order parameters $\phi$ and $g$; in particular with $g$. Of course, the $N \rightarrow \infty$ limit is mostly academic in the social context, since real social systems are small compared to thermodynamic systems.

\section{Nature of thermal society of agents}

Two basic questions come to mind when proposing a thermodynamic model for society, namely: what is the meaning of temperature and detailed balance? In the Axelrod model each agent is described as a vector whose $F$ components can take $q$ values that reflect the variety of postures an individual can have on a particular scope of action in society. Temperature may be thought as related to an energy scale that competes with the regular interactions between individuals; a high temperature renders their interactions moot, while a low temperature leads to a domination of the individual interactions and to the system settling into a minimum energy state. In this sense, in a society at high temperatures individuals would have fluctuating positions with a small relation to those of their circle of interaction, whereas at low temperatures individuals would pay much attention to their circle of interaction and tend to lower posture differences. In terms of a statistical ensemble approach, a group of individuals of the society would be subjected to a temperature reservoir that competes with individual interactions.

What sets this temperature scale might be any motive for confusion, for speculation or for uncertainty that could disrupt bonds between individuals, the agreement is not conducive to the benefit of the individual. This would be consistent with regarding temperature as a parameter coupled to entropic effects. In social terms, as the temperature increases the agents communicate less effectively, their coupling decreases, they are less convincing, in a sense, to their neighbors who choose or preserve their own positions without regard to their peers. This has entropic benefits since there is an increasing amount of ways people can disagree.

Thermal equilibrium is a situation where some Helmholtz-type free energy is minimal, and energy fluctuations exist subject to the condition of detailed balance. From Eq. (2) one can see that detailed balance dictates that an individual in a highly probable state $n$ should balance with an individual in a less probable state $m$. It is reasonable to expect that it is easier for the individual in state $m$ to adjust itself to the mainstream rather than the other way around. This can be seen as peer pressure or pressure to conform to the norm. This is an interesting perspective in the sense that varying 
temperature can yield thresholds for phase changes and set tipping points for collective behavior. On the other hand, a single temperature may not be set for different scopes of action, since a religious posture is certainly less fluctuating than a political posture. In our model this may be taken into account by weighting the interaction factor, $J_{i j}$, that depends on the cultural-featured $F$ vector.

\section{Summary}

We have presented a thermodynamic counterpart of the Axelrod model of social influence. The transfer matrix method was used to exactly solve for the thermodynamic and critical properties of the 1D model. A unifying proposal was made to compare exponents across different 1D discrete models, since current definitions depend on model details. We have also interpreted the implication of thermodynamic cultural dissemination.

The differences in internal symmetry and interaction in the thermodynamic Axelrod model with respect to the Potts model where not relevant from the point of view of criticality. An order-disorder phase transition occurs at $T=0$ independent of the cultural trait $q$ and feature $F$ variables. Our model, emulating Axelrod rules, increases the tendency to share cultural traits as the agents are more similar. This feature precipitates ordering in the system toward lower energy $(\mathrm{T}=0)$ as compared to Ising-like Hamiltonians. We expected that this feature would bring about absorbing-state type behavior, but in ring-like topologies fluctuations would be too strong so there would be only multicultural states for all finite $T$. Although the new Hamiltonian has a state-dependent bonding interaction, the model belongs to the Ising and Potts universality class.

The comparison with a Hamiltonian system sheds some light on the effects of two of the main ingredients of the out-of-equilibrium Axelrod model in the $1 \mathrm{D}$ case. The presence of mass media carries the system only to nonuniform regime. On the other hand, the thermodynamic counterpart also revealed that the original out-of-equilibrium 1-D Axelrod model in the limit $N \rightarrow$ $\infty$ remains in a disordered state for any finite noise, as expected for any 1D interacting particle system. It would be interesting to study the model in higher dimensions, where energy related effects can have an increasingly stronger role as compared to entropic features. 


\section{Acknowledgments}

Y.G. thanks support from the Venezuelan Government's project Misión Ciencia and the Instituto Venezolano de Investigaciones Científicas (IVIC). I.B. appreciates the financial assistance from IVIC through project No. 441. Y.G. is thankful for assistance from the Condensed Matter Laboratory at Universidad Simón Bolívar.

\section{References}

\section{References}

[1] R. Axelrod, J. Conflict Resolut. 41, 203 (1997).

[2] C. Castellano, S. Fortunato, V. Loreto, Rev. Mod. Phys. 81, 591 (2009).

[3] A. Barrat, M. Barthlemy, A. Vespignani, Dynamical Processes on Complex networks (Cambridge University Press, Cambridge, 2008).

[4] C. Castellano, M. Marsili, A. Vespignani, Phys. Rev. Lett. 85, 3536 (2000).

[5] J.C. Gonzalez-Avella, M.G. Cosenza, K. Tucci, Phys. Rev. E 72, 065102 (2005).

[6] J.C. Gonzalez-Avella, V.M. Eguiluz, M.G. Cosenza, K.Klemm, J.L. Herrera, M. San Miguel, Phys. Rev. E 73, 046119 (2006).

[7] Y. Gandica, A. Charmell, J. Villegas-Febres, I. Bonalde, Phys. Rev. E 84, 046109 (2011).

[8] K. Klemm, V. M. Eguíluz, R. Toral, M. San Miguel, J. Econ. Dyn. Control 29, 321 (2005).

[9] K. Klemm, V. M. Eguíluz, R. Toral, M. San Miguel, Phys. Rev. E 67, 045101 (2003).

[10] R. Toral, C. J. Tessoni, Commun. Comput. Phys. 2, 177 (2007).

[11] O. Al Hammal, H. Chaté, I. Dornic, M. A. Muñoz, Phys. Rev. Lett. 94, 230601 (2005). 
[12] B. J. West, E. Geneston, P. Grigolini, Phys. Rep. 468, 1-99 (2008).

[13] M. Henkel, M. Pleimling, Non equilibrium phase transitions Volume 2: Ageing and Dynamical Scaling far from equilibrium (Springer, Heidelberg, 2010).

[14] B. Diu, C. Guthmann, D. Lederer, B. Roulet, Physique Statistique (Hermann, Paris, 1989).

[15] M. Kardar, Statistical Physics of Fields (Cambridge University Press, Cambridge, 2007).

[16] R. K. Pathria, Statistical Mechanics (Butterworth-Heinemann, Oxford, 1996), 2nd ed.

[17] N. Goldenfeld, Lectures on Phase Transitions and the Renormalization Group (Perseus Books, Reading, 1992).

[18] Y. Gandica, Ph.D. thesis, Instituto Venezolano de Investigaciones Científicas (2012).

[19] K. Klemm, V. M. Eguíluz, R. Toral, M. San Miguel, Physica A 327, 1 (2003).

[20] L. R. Peres, J. F. J. Fontanari, J. Phys. A: Math. Theor. 43, 055003 (2010).

[21] J. C. Gonzalez-Avella, Ph.D. thesis, Universitat de les Illes Balears (2010). 